\title{
Analysis on Vocational Recognition of Aged Care Workers
}

\author{
Dapeng Cong \\ Yantai Nanshan University \\ Yantai, Shandong, China 265713 \\ Yan Gao \\ Yantai Nanshan University \\ Yantai, Shandong, China \\ Zongming Guo \\ Yantai Nanshan University \\ Yantai, Shandong, China
}

\author{
Shaojun Feng \\ Qilu Hospital of Shandong province Nanshan Branch \\ Yantai, Shandong, China \\ Shengqun Wang \\ Yantai Nanshan University \\ Yantai, Shandong, China \\ Haiming Zong \\ Yantai Nanshan University \\ Yantai, Shandong, China
}

\begin{abstract}
The article adopts the questionnaire method and interview method and does a research into care workers in pension institution from two aspects of professional identity and professional demand. Researches find that care workers in pension institution have a low professional identity. Their own occupational demands mainly include higher wages, the guaranty of welfare policy, law guaranty, making effort recognized and organizing training regularly. Finally the writer, under the perspective of social support, tries to make suggestions from the government level, social level and social work level. It will play a crucial role in improving care workers' sense of identity and enthusiasm of the service about their jobs. It also will make contributions to promoting social welfare and the development of old-age care.
\end{abstract}

Keywords-aged care workers; professional identity; occupational demand; policy

\section{INTRODUCTION}

Professional identity refers to a person who thinks his job is worthwhile from his heart and then will feel the pleasure from it. Care worker's identify of the work is influenced by various factors, such as working treatment, social status etc. Demission rate of kindergarten teachers abroad has been high at a time. Taking America as the example, in 1988-1992 70\% of teachers walked off the job due to the low income and social status. The reasons in care workers' identifications to their jobs, are mainly from the following several aspects.

\section{REASON ANALYSIS ON AGED CARE WORKERS' LOW PROFESSIONAL IDENTIFICATIONS}

\section{A. The Low Salary}

In our country, low salary is the significant cause that people are unwilling to be an aged care worker and stick on the nurse job. In the writer's research, the monthly pay (before tax) of $81.5 \%$ of workers ranges from RMB 2000 to RMB 3000 The writer has just talked about that $89.2 \%$ of the care workers' main considerations in choosing the pension nursing services as their jobs are wages and welfare treatment. But in the face of such treatment, local laid-off workers will not choose such jobs at all. The workers with a few years working experience will engage in family care workers or nannies and even divert. As is known to all, the nurses are very well paid and their treatments are several times higher than that of the care workers. Nurses are more professional to do the aged care work. But the fact is that they will not give up the high salaries for that job.

\section{B. Heavy Workload}

According to the survey, the contents of care workers in nursing home mainly include two: cares of daily life and cleaning of environmental health. Although there are only two above, all that they take care of are the olds who cannot care themselves meanwhile cannot do without them any time. Therefore, the amount of their work is really great. The writer finds that compared with nursing home, there are systematic written specifications in old people's home, which guide and manage the work of the care worker. Their daily work schedule is as follows.

- 6:00 Care workers shifting, duty officers starting to work.

- 6:30 Taking charge of the olds' dressing, going to the toilet, washing face, rinsing the mouth and fetching water.

- 7:30 Starting to have breakfast, delivering meal, feeding meal and milk.

- 8:20 Cleaning indoor sanitation. Medicine on time for the old man according to the advice of histher family 
and doctors. Nursing the old according to the provisions.

- 10:00 Assisting the olds in exercising, walking and doing recreational activities.

- 10:20 Starting to have patients diet, delivering meal and feeding.

- 11:20 Starting to have lunch, delivering meal and feeding.

- 12:00 Having a rest.

- 13:30 Fetching water, doing some cleaning taking good daily care of the olds.

- 15:00 Organizing the olds to do activities.

- 16:10 Starting to have patients diet, delivering meal and feeding.

- 17:00 Starting to have dinner, delivering meal and feeding. Duty officers looking after the olds and making records for the olds' health that day.

- 19:50 Care workers shifting, duty officers starting to work.

- 20:00 After duty officers taking over from care workers, then taking care of the olds until the next morning at8:00 according to the specified service contents.

So we can see that the contents covering in their jobs are very miscellaneous and toilsome. It includes the cares of daily life, medical nursing services, culture and entertainment, physical fitness activities and the cleaning of environmental health, etc. Their daily work contents are embedded into a set of standard system in chronological order and should not be changed.

\section{Great Psychological Pressure}

In the face of such heavy works, various psychological problems will likely appear on care workers. The labour intensities of their works are very great because one care worker will care for several old people's daily life. Accidents, such as choked, tumbling and fracture, etc. will happen if there is any carelessness. These mistakes and accidents will have deadly impact on the olds who especially is senile or seriously ill. For this reason, they must take a cautious attitude all the time so it is likely to lead to excessive fatigue in their bodies and minds. Moreover, what they do are usually dirty and they always feel tired about it such as handling with urination and defecation of the olds who are incontinent and constipated and other chores. Their efforts are disproportional to the payment, so it is likely to cause psychological imbalance and bring negative feelings to them.

\section{ANALYSIS ON OCCUPATIONAL DEMAND OF CARE WORKERS}

According to Maslow Model, people want not only to survive but also to enrich life. They also want to live happily and make social harmony. That is to say, a good life also depends on the self-actualization of people's own value and its function to society. Physiologically, it is necessary to provide care workers with food, clothing and shelter which are their most basic needs. During the nursing services, society firstly should ensure that the resources for satisfying care workers' primary physiological needs are available to be utilized. The needs for care workers' safety include economic safety and personal safety, and that is to say that care workers should feel that their basic living and circumstances are fully ensured. After having the primary living security, care workers will desire to communicate more with their families and their friends, all of which are the social communicating activities and social needs they are eager for. Besides that, care workers also have needs for professional knowledge like others. Because of the rapidly developing science and technology and the rapidly changing social culture, learning has become lifelong gradually and it also as the premise ensures that they will be adapted to the development of society. The group consisting in aged care workers has been become an important part of society nowadays and is the core and pillar of pension nursing industry. The future of pension nursing industry also depends on its development. So we must attach importance to this group. By knowing and concerning with their various needs, more people will be attracted to join in this team in order to make the team more stable and increasingly expend.

\section{A. Wages Increase}

The writers has mentioned ahead of the article that the low salary is the main and primary one of the reasons that care workers are not satisfied with the work condition. Most of their wages will be sent home by post while only a little part will be left to buy some daily supplies for themselves. Although in the resourceful society, the low wages also limit them in obtaining and using the resources. Their increasing needs for resources are not satisfied and this will lead to increasing their inner fall and needing strongly for it psychologically. Consequently, the high wages have become the urgent demand of the care workers. According to the survey, it shows that $43.1 \%$ of the care workers hope that their wages will be in RMB 3000 to RMB 4000 and $38.5 \%$ hopes wages in RMB 4000 to RMB 5000 . Faced with the development of this industry, a lot of care workers will leave if the salary is still in present level. Then this industry will not develop any longer. There is no native worker in these two institutions, which precisely reflects a phenomenon that few of 4050 native people who have laid off or at home without job is hardly willing to engage in this industry. The writer thinks that this group is one of the important potential resources for developing nursing industry. If this resource can be used effectively, not only the employment but also the development of nursing industry will be promoted. So the high wages will make contributions to the stable development of the nursing team now and meanwhile attract the 4050 native people to join in this industry so that human resources will get effective configuration.

\section{B. The Guaranty of Welfare Policy}

For treatment, except increasing the urgent demand of wages, the issue on demand to social security is still comparatively prominent. According to the provisions of Labour Law of the People's Republic of China, it is a duty of retirement organizations to buy the social insurance for the 
aged care workers. Only in this way, they will have a sense of security that their present and future life are ensured. However the fact is antipodal. Care workers cannot enjoy this treatment because most of the retirement organizations do not do so. Actually it virtually reduces their benefits. Many care workers express their concerns about the future life: Without the social security, they will not obtain the sources of livelihood and fall into various difficulties. According to the writer's survey, no nursing home carries out the duty to buy social insurance for the aged care workers by. Only three kinds of social insurance are bought to care workers under the age of 40 in old people's home. The reasons are mainly caused in two aspects: One is the plight of the retirement organizations. Organizations will increase their labor costs if the social insurance of their workers is bought. The head of the two institutions all reflect to writer that the charges by retirement organizations are lower while the operation costs are higher now, which will cut profits. So there is not enough money for care workers to buy social insurance. The other is care workers themselves. They are unwilling to pay the individual pay part of the whole social insurance.

\section{Law Guaranty}

Because the education background of the care workers is low and they have not received a good education, their concept and awareness of law is relatively weak. Their shortage of basic legal knowledge leads to the weak self-protection. For some certain problems, there is no one for them to talk about. No correct guidance will lead to their loss of the right direction and the rights. They are a disadvantaged group in society. All people are equal before the law. They should have either the duty to fulfill the obligations or the guarantee to enjoy the rights. Society lacks of attention to the aged care workers and always ignores their due rights. Consequently, only in the condition that the workers have studied and understood the laws and abided by them can the due rights be obtained to better safeguard their personal interests.

\section{Making Effort Recognized}

At present, the work of aged workers have not been enough socially recognized and respected yet. People always have a stereotype about care workers. A stereotype namely is an impression on a social group formed by the people. A stereotype about a certain group is composed of positive and negative characteristics. It may be correct and may be wrong, too. The disadvantages of stereotype are as follows: One is that it exaggerates the similarity among the members of the group thus to leads to the preconceived and incomplete deviations to individual aesthesia; and two is that it exaggerates the diversity among the groups. It will be likely to cause prejudice and discrimination. While people still have prejudice and discrimination to the care workers. It will leads to low social recognition. Although in some special holidays, government leaders or social person, etc will go to retirement organizations to comfort the elderly and care workers, the retirement organizations have not aroused much wider social concern, support and respect. They generally feel their low social status and without understanding and recognition, facing the bias from different groups of people.
Through the interview, we can know that care workers often feel inferior to their own work and weak professional pride. They rarely feel the atmosphere of outside support for their work. However there are some social supports and volunteer services occasionally, but it is very optional. Care workers are still in lack of long-term stable support source and supporting way.

\section{E. Organizing to Train Regularly}

Government should attach to the security problems of care workers and strengthen the efforts to social insurance. It also should pay attention to exploring various ways about the social insurance of care workers proactively to establish a perfect social security mechanism gradually. Consequently after eliminating their worries, the development of pension services industry could be promoted truly and effectively.

\section{CONCLUSION}

Social supports of care workers are the support systems coprovided by the formal and informal systems of organization, government and society, etc and they are playing a different role. Social support affects the mental health, job content and job satisfaction of care workers. Improving the level of social support can be able to satisfy the needs of the care workers. So the writer puts forward some views and suggestions on how to further meet the needs of care workers from the perspective of social support.

At this stage the country is in the transition from the management government in the past to the service oriented government. Government transfers the way mainly based on management and to build a service-oriented way by means of updating the governing concept and transforming the government function. By means of function of public service to formulate and implement the public policy, government also should provide support and protection for the aged care workers thus to maintain public order and promote social fairness and protect the rights and interests of care workers.

\section{A. Increasing the Investments and Improving Welfares}

In recent years, although governments at all levels constantly increase investment in public utilities, it is not compatible with the investment to retirement organizations and the level of economic and social development. Low wages and benefits lead to the rising increased mobility of care workers, and many retirement organizations reflect not being able to retain the care workers any longer. Therefore, governments at all levels should start from performing the functions of social management and public service and regard the pension industry as the focus of the construction of social security system. The attention to care workers also should be increased. Firstly, government should further improve regulations and system construction of the relevant pension institutions and support institutions for the pension industry by formulating the corresponding institutional pension policy to give the corresponding financial support and tax incentives, etc. Secondly, government should actively encourage social forces to set up pension institutions and provide strong support for it such as one-off construction subsidies and regular beds 
subsidies. It can alleviate the construction and operating pressure of the pension institutions and contribute to improving the treatment of care workers. Thirdly, care worker position can be classified as public welfare position and give them appropriate subsidies. Meanwhile, government also can provide appropriate subsidies of special talents to the care workers in order to attract and retain talents.

\section{B. Strengthening the Safeguard Mechanisms}

At the same time of seeking the way to improve the incomes of the care workers, it is also important to strengthen their right protection. Only in this way would their work be more stable and they would focus more on working. But at present, the social security system of retirement organization is not completely established. Care workers, according to Labor Law, should have the right of rest, vacation and labor hygiene protection, etc. It is only a short time that care worker has listed in Industry Category and Occupational Classification issued by our country. So there is still in lack of a set of perfect legal basis in wages, social security, etc. For care workers, there is no legal working hours and formal rest system, which would make them more fatigue; but due to historical reasons, non-government organizations in our country develop slowly. Some organizations often accompanied by official factors and it is difficult to meet the needs of social diversity and to effectively help the social vulnerable groups. So government should formulate relevant policies to protect and promote the development of the non-profit organization and make the corresponding supervision and management at the same time. The non-profit organizations will play a more positive role in the social support of the aged care workers through continuously improving its construction.

\section{REFERENCES}

[1] Liu Shenmei, Long Anguo, Zhuang Huaying, Zhou Liping, Tang Xiaoli. Multivariate Analysis on the Influences of the Characteristics of Aged Care Workers to The Physical and Mental Health of the Old People in Retirement Organizations. [J]. Attend to Practice and Research. 2013 (13).

[2] Xiao Yu, Wu Xianhe. The Research Progress of Aging of Population and Aged Care. [J]. Journal of North Pharmacy. 2013 (03).

[3] Li Jie, $\mathrm{Cu}$ Guihua, Jiang Rongrong, Jiang Gaoxia. Present Situation of Aged Care Services Staff in China and Outlook on Personnel Training. [J].Journal of Nanjing University Of Chinese Medicine (Social Sciences). 2012 (04).

[4] An Libin, Li Wentao, Xie Shuhong, Zhou Xiaohua. Sustainable Development on Aged Care under the Background of China's Ageing Population. [J]. Chinese Journal of Gerontology. 2012 (22)

[5] Chen Kaimei. Analysis on Supply and Demand Structure of Aged Care Staff in Pearl River Delta Cities. [J]. Economic Forum. 2012 (11).

[6] Huang Fei, Zhang Huijun, Xie Jiemei. Research Progress of the Training on Aged Care Staff in China. [J].Nursing Research. 2011 (03).

[7] Tao Weiwei, Zhang Xinyu, Yi Yunling, Sun Jing, Zhang Hong. Cognition and Demand of Aged Care Staff in Home Care Service Center to Aged Care Knowledge. [J]. Chinese Journal of Nursing Education. 2010 (10).

[8] Xue Yanqing. Theory Source of the Plurality of the Subject for Public Service Supply. [J]. Lanzhou Academic Journal. 2010 (05).

[9] Chen Zhuoyi, Chen Weiran. Current Situation and Countermeasures of the Team Construction of Aged Care Staff in China. [J]. Journal of Changsha District Vocational Technical College. 2009 (04). 